\title{
LONG-WAVELENGTH EXCESSES IN TWO HIGHLY OBSCURED HIGH-MASS X-RAY BINARIES: IGR J16318-4848 AND GX 301-2
}

\author{
D. L. KaPlan, ${ }^{1,2}$ D.-S. MoON, ${ }^{3,4,5,6}$ AND W. T. ReACH ${ }^{7}$ \\ Received 2006 June 15; accepted 2006 August 14; published 2006 September 13
}

\begin{abstract}
We present evidence for excess long-wavelength emission from two high-mass X-ray binaries, IGR J16318-4848 and GX 301-2, that show enormous obscuration $\left(N_{\mathrm{H}} \simeq 10^{23}-10^{24} \mathrm{~cm}^{-2}\right)$ in their X-ray spectra. Using archival near- and mid-infrared data, we show that the spectral energy distributions of IGR J16318-4848 and GX 301-2 are substantially higher in the mid-infrared than their expected stellar emission. We successfully fit the excesses with $\sim 1000 \mathrm{~K}$ blackbodies, which suggests that they are due to warm circumstellar dust that also gives rise to the $\mathrm{X}$-ray absorption. However, we need further observations to constrain the detailed properties of the excesses. This discovery highlights the importance of mid-infrared observations for understanding highly obscured X-ray binaries.

Subject headings: circumstellar matter — infrared: stars — stars: emission-line, Be - X-rays: binaries X-rays: individual (GX 301-2, IGR J16318-4848)

Online material: color figures
\end{abstract}

\section{INTRODUCTION}

Recently a growing number of hard X-ray sources have been discovered by the IBIS-ISGRI soft gamma-ray detector aboard the INTEGRAL satellite (Winkler et al. 2003). These new sources show largely power-law spectra in the $\sim 15-60 \mathrm{keV}$ range, but they are defined by the very large column densities of intervening hydrogen atoms implied by spectral fits: $N_{\mathrm{H}} \geq$ $10^{23} \mathrm{~cm}^{-2}$ (and sometimes greater than $10^{24} \mathrm{~cm}^{-2}$; e.g., Revnivtsev 2003; Revnivtsev et al. 2003a, 2003b; Walter et al. 2003; Patel et al. 2004 and references therein), which corresponds to visual extinctions $A_{V}>100$ (using the usual relation of $A_{V}=N_{\mathrm{H}} / 1.8 \times 10^{21} \mathrm{~cm}^{-2}$; Predehl \& Schmitt 1995). Most of these sources show short-term variability, indicating their compact nature, and some of them have in fact been detected with coherent intensity modulations that are suggestive of the existence of neutron stars or white dwarfs (e.g., Lutovinov et al. 2005). The extremely large absorption has probably hidden the full extent of the population from previous X-ray missions that lacked sufficient hard X-ray sensitivity, as in the soft Xray range $(\leq 2 \mathrm{keV})$ they are quite faint even with the sensitive Chandra and XMM-Newton satellites.

One of the first and most extreme of these sources is IGR J16318-4848, discovered on 2003 January 29 (Courvoisier et al. 2003). Continued monitoring with INTEGRAL measured a mean flux of $\sim 6 \times 10^{-11} \mathrm{ergs} \mathrm{cm}^{-2} \mathrm{~s}^{-1}$ in the $20-50 \mathrm{keV}$ energy range and found variability on timescales $\gtrsim 1000 \mathrm{~s}$ (Walter et al. 2003), while a subsequent XMM-Newton observation revealed a rich array of X-ray emission lines on top of the absorbed powerlaw continuum (Matt \& Guainazzi 2003; Walter et al. 2003). One of the most noticeable features from the X-ray spectra of

\footnotetext{
${ }^{1}$ Kavli Institute for Astrophysics and Space Research, Massachusetts Institute of Technology, 77 Massachusetts Avenue, 37-664H, Cambridge, MA 02139; dlk@space.mit.edu.

2 Pappalardo Fellow.

${ }^{3}$ Division of Physics, Mathematics, and Astronomy, California Institute of Technology, MC 103-33, Pasadena, CA 91125; moon@srl.caltech.edu.

${ }^{4}$ Robert A. Millikan Fellow.

${ }^{5}$ Space Radiation Laboratory, California Institute of Technology, MC 22047, Pasadena, CA 91125.

${ }^{6}$ Department of Astronomy and Astrophysics, University of Toronto, Toronto, ON M5S3H8, Canada.

${ }^{7}$ Spitzer Science Center, Infrared Processing and Analysis Center, California Institute of Technology, MC 220-6, Pasadena, CA 91125; reach@ipac.caltech.edu.
}

IGR J16318-4848 is the extremely high column density: $N_{\mathrm{H}} \simeq 2 \times 10^{24} \mathrm{~cm}^{-2}$. Accurate localization from XMM-Newton made it possible to identify a bright Two Micron All Sky Survey (2MASS; Skrutskie et al. 2006) star as its near-infrared (nearIR) companion (Foschini et al. 2003; Walter et al. 2003). The companion is a $\mathrm{sgB}[\mathrm{e}] \mathrm{star}$ (a B supergiant that shows forbidden emission lines; see Lamers et al. 1998) with many emission lines in the near-IR (Filliatre \& Chaty 2004). The optical and nearIR data favor placing the counterpart at a distance of 0.9-6.2 kpc with $A_{V} \simeq 17$ mag (Filliatre \& Chaty 2004), and overall the near-IR spectrum of IGR J16318-4848 is similar to that of CI Cam, the $\mathrm{sgB}[\mathrm{e}]$ companion of the highly obscured Galactic Xray binary XTE J0421+560 (Wagner \& Starrfield 1998; Clark et al. 1999; Belloni et al. 1999; Boirin et al. 2002).

Revnivtsev et al. (2003b) and Filliatre \& Chaty (2004) suggested that IGR J16318-4848 is a high-mass X-ray binary (HMXB) surrounded by dense circumstellar material and powered by accretion from a stellar wind. The huge difference in the absorption between the X-ray $\left(N_{\mathrm{H}}=2 \times 10^{24} \mathrm{~cm}^{-2}\right.$ implies $A_{V}=1100 \mathrm{mag}$ ) and near-IR suggests that much of the $\mathrm{X}$-ray absorption is intrinsic to the compact object (Revnivtsev et al. 2003b; Filliatre \& Chaty 2004) and is in fact due to the wind of the $\operatorname{sgB}[e]$ companion accreting onto it. Based on the similarity of IGR J16318-4848 to XTE J0421+560/CI Cam, where a substantial circumstellar dust shell was revealed by IRAS data in the 12-100 $\mu \mathrm{m}$ range (Belloni et al. 1999; Clark et al. 1999), Filliatre \& Chaty (2004) predicted the existence of dust in the IGR J16318-4848 system, although they were not able to confirm its existence.

GX 301-2 is another high-mass X-ray binary with the early Be-type supergiant (or hypergiant) optical companion Wray 977 (Kaper et al. 1995), and as such may resemble IGR J16318-4848, although there are some differences. As with IGR J16318-4848 and XTE J0421+560, the X-ray spectrum of GX 301-2 is characterized by variable strong photoabsorption $\left(N_{\mathrm{H}} \simeq 10^{23}-10^{24} \mathrm{~cm}^{-2}\right.$, compared with $A_{V} \approx 6$; Vidal 1973) with fluorescent iron lines (Mukherjee \& Paul 2004), and the optical spectrum is very rich in emission lines from hydrogen and other elements. Scaling its optical luminosity to that of the similar star $\zeta^{1}$ Sco, Kaper et al. (1995) determined a distance of $5.3 \mathrm{kpc}$ and an X-ray luminosity of $\sim 10^{37} \mathrm{ergs} \mathrm{s}^{-1}$ for the system.

Based on the similarities, especially the heavy X-ray obscura- 
TABLE 1

INFRARED PHOTOMETRY OF IGR J16318-4848 AND GX 301-2

\begin{tabular}{|c|c|c|c|c|c|c|c|c|c|c|}
\hline \multirow[b]{2}{*}{ Source } & \multicolumn{3}{|c|}{ 2MASS (mag) } & \multicolumn{4}{|c|}{ IRAC FLux (Jy) } & \multicolumn{3}{|c|}{ MSX Flux (Jy) } \\
\hline & $\begin{array}{c}J \\
(1.24 \mu \mathrm{m})\end{array}$ & $\begin{array}{c}H \\
(1.66 \mu \mathrm{m})\end{array}$ & $\begin{array}{c}K_{s} \\
(2.16 \mu \mathrm{m})\end{array}$ & $\begin{array}{l}\text { IRAC-1 } \\
(3.6 \mu \mathrm{m})\end{array}$ & $\begin{array}{l}\text { IRAC-2 } \\
(4.5 \mu \mathrm{m})\end{array}$ & $\begin{array}{l}\text { IRAC-3 } \\
(5.8 \mu \mathrm{m})\end{array}$ & $\begin{array}{l}\text { IRAC-4 } \\
(8.0 \mu \mathrm{m})\end{array}$ & $\begin{array}{c}M S X-\mathrm{A} \\
(8.28 \mu \mathrm{m})\end{array}$ & $\begin{array}{c}M S X-\mathrm{C} \\
(12.13 \mu \mathrm{m})\end{array}$ & $\begin{array}{c}M S X-\mathrm{D} \\
(14.65 \mu \mathrm{m})\end{array}$ \\
\hline IGR J16318-4848 ...... & $10.16(2)$ & $8.33(4)$ & $7.19(2)$ & $1.17(11)$ & $1.07(11)$ & $0.814(8)$ & $0.64(6)$ & $0.48(5)$ & & \\
\hline
\end{tabular}

NoTES. - Values in parentheses are $1 \sigma$ statistical uncertainties, in mag or Jy as appropriate. We added 2\% zero-point uncertainties to the 2MASS data, following CWM03.

tion, among these three sources, it seems promising to search for emission from the circumstellar dust that would be responsible for their heavy obscuration. In this Letter, we report the discovery of evidence for such emission in the infrared spectral energy distributions (SEDs) of IGR J16318-4848 and GX 301-2.

\section{ARCHIVAL INFRARED DATA}

Table 1 lists the IR photometric data for IGR J16318-4848 and GX 301-2. The near-IR magnitudes are from the 2MASS point-source catalog, where the counterparts of the two sources are 2MASS J16314831-4849005 and J12263756-6246132 for IGR J16318-4848 and GX 301-2, respectively.

For the mid-IR fluxes, we analyzed archival data from the Galactic Legacy Infrared Mid-Plane Survey Extraordinaire (GLIMPSE; see Benjamin et al. 2003 for a detailed description), which is one of the Legacy projects of the Spitzer Space Telescope (Werner et al. 2004). The GLIMPSE survey covers the Galactic plane region $(|l|,|b|)=\left(10^{\circ}-65^{\circ}, \leq 1^{\circ}\right)$, including the positions of two sources, with the Infrared Array Camera (IRAC; Fazio et al. 2004) at the four mid-IR wavelengths 3.6, 4.5, 5.8, and $8.0 \mu \mathrm{m}$. The spatial resolution of the IRAC data is $2^{\prime \prime}$, with 1 1.2 pixels. We used the individual basic calibrated data of the GLIMPSE survey, and the program $\operatorname{IDs}^{8}$ for the data sets we used in this Letter are 191 for IGR J16318-4848 and 189 for GX 301-2. For both sources the central pixels were saturated in the 3.6 and $4.5 \mu \mathrm{m}$ images, so we measured the flux using routines developed by a staff member at the Spitzer Science Center for this specific problem. ${ }^{9}$ Briefly, we fit point-spread functions to the unsaturated wings of the sources, replaced the saturated pixels by the scaled point-spread function, and then summed all of the pixels. To account for potential calibration problems involved in this process, we increased the uncertainties for the measured fluxes to $10 \%$, which we believe are conservative limits. We give our corrected photometry in Table 1.

The mid-IR fluxes of the counterparts to IGR J16318-4848 (Foschini et al. 2003) and GX 301-2 were also listed in the Midcourse Space Experiment (MSX; Price et al. 2001) ${ }^{10}$ Point

\footnotetext{
${ }^{8}$ See http://ssc.spitzer.caltech.edu/archanaly/status.

${ }^{9}$ See http://spider.ipac.caltech.edu/staff/jarrett/irac/method, and this program will be uploaded to http://ssc.spitzer.caltech.edu/archanaly/contributed as contributed software.

${ }^{10}$ See http://irsa.ipac.caltech.edu/Missions/msx.html.
}

Source Catalog (MSX6C). ${ }^{11}$ For IGR J16318-4848, the source MSX6C G335.6164-00.4479 is 2" away from the near-IR counterpart (comparable to the $M S X$ position uncertainty) and is only detected in the MSX A band (centered at $8.28 \mu \mathrm{m}$ and covering 6.8-10.8 $\mu \mathrm{m}$ ) with a flux consistent with although somewhat lower than that of the IRAC data at $8 \mu \mathrm{m}$. For GX 301-2, the source MSX6C G300.0980-00.0351 is 0.4 away from the near-IR counterpart and is detected in the A, C (centered at $12.13 \mu \mathrm{m}$ and covering $11.1-13.2 \mu \mathrm{m}$ ), and D (centered at $14.65 \mu \mathrm{m}$ and covering $13.5-15.9 \mu \mathrm{m}$ ) bands. Again, like IGR J16318-4848, the A-band flux of GX 301-2 is somewhat lower than the corresponding GLIMPSE IRAC flux at $8 \mu \mathrm{m}$. The lower fluxes of the both sources at the MSX A band may be caused by the broad absorption feature of silicates around $10 \mu \mathrm{m}$ in the interstellar medium (Draine 2003) visible only in the MSX A band because of its longer wavelength coverage.

\section{FITTING THE SPECTRAL ENERGY DISTRIBUTION}

We fit model stellar spectra to the infrared SEDs of IGR J16318-4848 and GX 301-2, using the extinction law of Indebetouw et al. (2005); this empirical model is a smooth function only and does not include the effects of the silicate features discussed above, and so the detailed fitting right around $10 \mu \mathrm{m}$ (i.e., near the $M S X$ A band) may be suspect. However, its simplicity and the fact that it was derived from 2MASS and IRAC data (such as those we are considering here) make it acceptable. When comparing the model to the data, we integrated the model flux over the filter bandpasses for $2 \mathrm{MASS}^{12}$ and IRAC ${ }^{13}$ and with appropriate Gaussians for $M S X$; we also added 2\% zero-point uncertainties for the 2MASS data (Cohen et al. 2003, hereafter CWM03) since the statistical uncertainties were so small. The basic stellar models were those of B0 I stars (with effective temperature 26,000 K), consistent with previous fits and spectroscopy of these objects (see Table 2; Kaper et al. 1995; Filliatre \& Chaty 2004). Our stellar models were from the Kurucz (1993) ATLAS9 grid, with solar metallicity and a microturbulent velocity of $2 \mathrm{~km} \mathrm{~s}^{-1}$ (the $\mathrm{fp} 00 \mathrm{k} 2$ models). The stellar models are not very important for this application, since the stars are very nearly Rayleigh-Jeans tails

\footnotetext{
${ }^{11}$ Vizier Online Data Catalog, 5114 (M. P. Egan et al., 2001).

${ }^{12}$ See http://www.ipac.caltech.edu/2mass/releases/allsky/doc/sec6_4a.html.

${ }^{13}$ See http://ssc.spitzer.caltech.edu/irac/spectral_response.html.
}

TABLE 2

SED Fit Parameters and Near- and Mid-IR Fluxes of Highly Obscured Binaries

\begin{tabular}{|c|c|c|c|c|c|c|c|}
\hline SOURCE & Stellar TyPE & $\begin{array}{c}A_{V} \\
\text { (mag) }\end{array}$ & $\begin{array}{l}T_{\text {dust }} \\
(\mathrm{K})\end{array}$ & $R_{\text {dust }} / R_{\text {star }}$ & $\begin{array}{c}F_{\nu, \text { dust }}(8 \mu \mathrm{m}) \\
(\mathrm{mJy})\end{array}$ & $\begin{array}{c}\chi^{2} / \text { dof, } \\
\text { Star }^{\mathrm{a}}\end{array}$ & $\begin{array}{c}\chi^{2} / \text { dof } \\
\text { Star+Dust }\end{array}$ \\
\hline IGR J16318-4848 ..... & B0 I & 18.5 & 1030 & 10 & 810 & 152 & $15 / 3$ \\
\hline GX $301-2 \ldots \ldots \ldots \ldots$ & B0 I & 7.1 & 740 & 9 & 440 & 121 & $6 / 5$ \\
\hline
\end{tabular}

Notes. - Dust model is a blackbody with the temperature given by $T_{\text {dust }}$ and a radius given as a multiple of the stellar radius. For convenience, we also provide the unabsorbed flux from the dust model at $8 \mu \mathrm{m}$.

${ }^{\text {a }}$ We do not give the degrees of freedom (dof) for the star, as the model was fitted to just the 2MASS data, while the $\chi^{2}$ is from all of the data in Table 1 . 

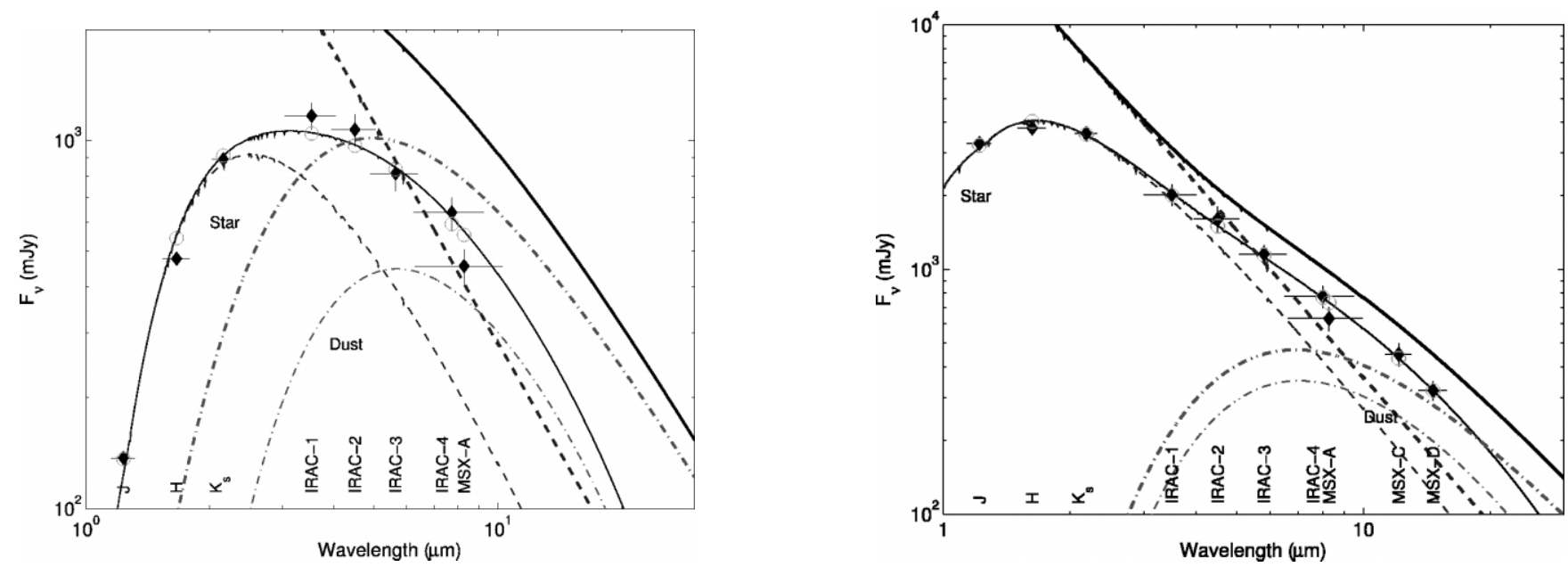

FIG. 1.-Fits to the SEDs of IGR J16318-4848 (left) and GX 301-2 (right). The fits show the model stellar photosphere (a B0 I model) and a cool blackbody representing the contribution of dust, all corrected for extinction. The data points are the diamonds, while the open circles are the model integrated through the filter responses. The thick lines show the star (dashed line), dust (dot-dashed line), and total (solid line) as corrected for absorption, while the thin lines show the same components without any correction. [See the electronic edition of the Journal for a color version of this figure.]

over the wavelength range in question $(>1 \mu \mathrm{m})$, but they did not complicate the fitting. For IGR J16318-4848, the effective temperature of the star is not precisely known, and the specific choice was largely a matter of convenience, but variations in the effective temperature between 15,000 and $34,000 \mathrm{~K}$ only change $A_{V}$ by $\pm 0.5 \mathrm{mag}$. We used the radii, gravities, and effective temperatures listed for the appropriate stellar type in Cox (2000, p. 389) ${ }^{14}$ although we in fact only used the radii for rough estimates since the luminosities of such massive and active stars can vary widely (factor of $\gtrsim 2$ variations in radius for a given effective temperature; Lamers et al. 1998).

We first determined the best-fit values of the extinction $\left(A_{V}\right.$; see Table 2$)$ with only the near-IR (2MASS) data because of the apparent mid-IR excess emission in the observed SEDs of both sources (see below); the values that we find are reasonably consistent (especially given the degeneracies between effective temperature and reddening at these wavelengths; see Filliatre \& Chaty 2004) with values from the literature. When we extended the fits to longer wavelengths, we immediately found that the stellar model alone could not fit the observed SEDs reasonably (see $\chi^{2}$ for the star alone in Table 2); rather, we found emission in excess of the stellar model at $\lambda \gtrsim 4 \mu \mathrm{m}$. In comparison, we have carried out SED fits of a number of other stars using 2MASS and GLIMPSE data, and see no excesses in the majority of the cases. We therefore concluded that the excess mid-IR emission is real and fit it with a 500-1500 K blackbody component representing dust emission; by using a blackbody, we do not mean to imply that the dust is optically thick, but are merely choosing a simple parameterization that roughly reproduces the shape of the observed emission. Although it is possible in principle that the presence of many emission lines in the near- or mid-IR can change the SEDs significantly, the success in fitting broadband photometry of CI Cam with just a stellar plus continuum model (Belloni et al. 1999) and the extremely large amplitude and wavelength range of the excesses make this unlikely. Table 2 and Figure 1 present the best-fit parameters and the results of the fits, respectively. In Table 2 we only give the radius as a multiple of the stellar radius since the distances of the two sources are not well determined, especially for IGR J16318-4848. As seen in

${ }^{14}$ Also see http://www.stsci.edu/hst/observatory/cdbs/k93models.html.
Figure 1, both objects are reasonably well fit by dust excesses with similar temperatures of $700-1000 \mathrm{~K}$ and with radii $\approx 10$ times the stellar radii, despite the drastically different observed SEDs (the observed differences are mostly due to different levels of extinction). Uncertainties in the effective temperature and hence extinction of the stars do not greatly affect the magnitude of the excess, but can alter $T_{\text {dust }}$ by $\pm 200 \mathrm{~K}$. The reduced $\chi^{2}$ value for IGR J16318-4848 with the dust excess is not especially low, but it is a significant improvement on the staronly model, and $60 \%$ of the $\chi^{2}$ comes only from the $H$-band point, which is primarily from the stellar spectrum; a more complicated dust model (see below) would likely improve the fit overall, but the current data do not warrant such a fit.

\section{DISCUSSION AND CONCLUSIONS}

Overall, the excess emission that we see appears consistent with that from warm dust, which is quite similar to the XTE J0421 + 560/CI Cam system (Belloni et al. 1999). However, we also investigated the possibility that the mid-IR excess of IGR J16318-4848 and GX 301-2 may come from free-free emission in the winds of the companions (see, e.g., Coté \& Waters 1987). Such emission originating from the stellar winds has certainly been detected in the radio, far-IR, and mid-IR spectra of a number of high-mass (but usually main-sequence, not supergiant) stars that are undergoing slow mass loss (Wright \& Barlow 1975). We found, however, that the free-free emission is not able to account for either the shape (see Fig. 2) or the magnitude of the excess emission that we see.

While we therefore believe that we have detected the presence of dust in these systems, our current data cannot strongly constrain the properties of the dust (total mass, and distributions of temperature and density), as the excess is only visible in a few data points and there could be substantial amounts of cooler dust at larger radii from the compact object that emit outside the observed band. While the fact that there is an excess is certain, the exact form is unknown. We have used cool blackbodies, which represent the data well. More realistic models, like a blackbody with modified opacity (Hildebrand et al. 1977; Dale et al. 2001) or the optically thin dust cloud (Waters et al. 1988) used by Belloni et al. (1999) are too unconstrained, although they can be easily manipulated into the forms of the observed excesses. 

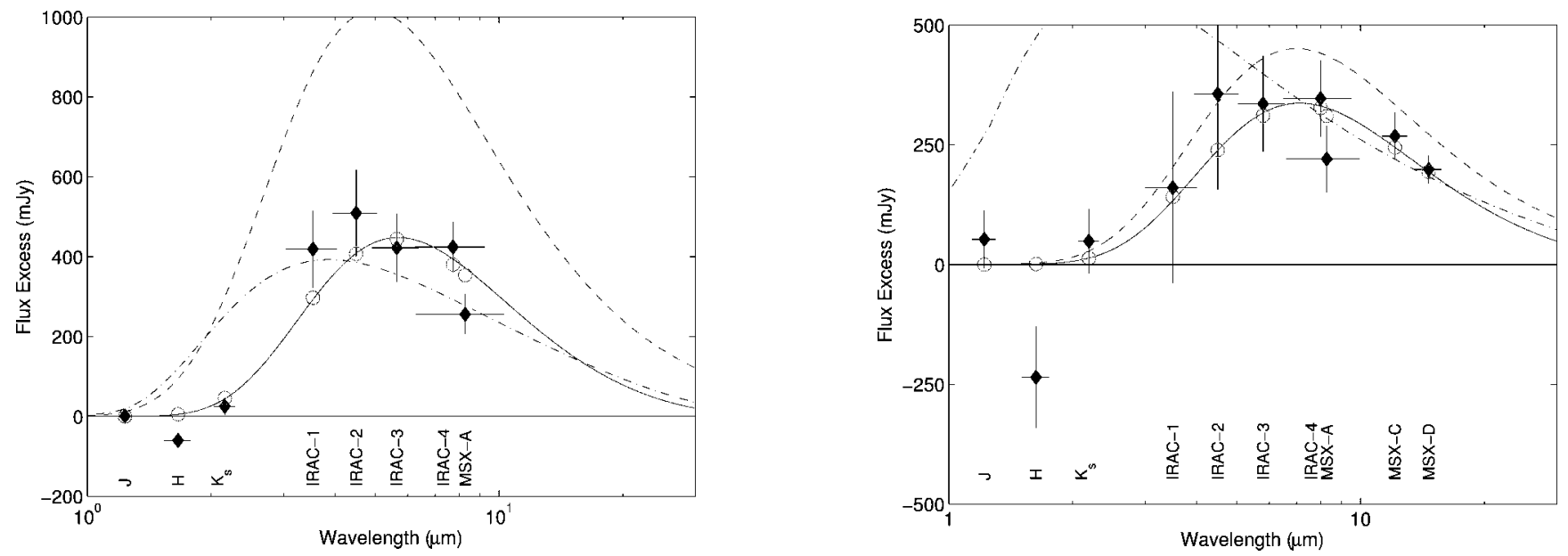

FIG. 2.-Flux excesses for IGR J16318-4848 (left) and GX 301-2 (right), compared to the stellar models in Fig. 1 and Table 2. The data points are the diamonds, while the open circles are the model integrated through the filter responses. The solid lines are the dust models (blackbodies) corrected for absorption, while the dashed lines are the dust models without absorption. The dot-dashed lines show something like the expected spectrum for free-free emission: power laws with $F_{\nu} \propto \nu^{0.8}$ (Wright \& Barlow 1975). [See the electronic edition of the Journal for a color version of this figure.]

Assuming that the emission is optically thin (without meaningful size estimates, we cannot verify this assumption), we can estimate the dust masses. Following Hildebrand et al. (1977), the dust mass visible at $8 \mu \mathrm{m}$ is $M_{\text {dust }}=F_{\nu} d^{2} / \kappa_{\nu} B_{\nu}\left(T_{\text {dust }}\right)$, where $d=5 d_{5} \mathrm{kpc}$ is the distance to the source and $\kappa_{\nu}=739 \mathrm{~cm}^{2} \mathrm{~g}^{-1}$ is the dust opacity at $8 \mu \mathrm{m}^{15}$ (which we take from Li \& Draine 2001, and which is proper only for the interstellar medium). This gives us $M_{\text {dust }} \geq 1 \times 10^{-8} M_{\odot} d_{5}^{2}$ for both IGR J16318-4848 and GX $301-2$. These amounts are significantly less than the $\geq 0.002 M_{\odot}$ of dust that Belloni et al. (1999) found around CI Cam, but our values are lower limits because we assumed a single-temperature optically thin case.

In summary, by comparing the near- and mid-IR data for IGR J16318-4848 and GX 301-2 to models of stellar photospheres, we have shown strong evidence for mid-IR excesses. In the absence of other plausible models, we believe that this excess is likely due to circumstellar dust emission. To the best of our knowledge, this makes IGR J16318-4848 and GX 301-2 the second

\footnotetext{
${ }^{15}$ Since we are modeling the dust with a blackbody distribution, the mass estimates at other wavelengths will be similar, and should vary only due to the non-gray opacity. Over the band where we have data (3 to 10 or $15 \mu \mathrm{m}$ ), the opacity changes by $<50 \%$, except for near $10 \mu \mathrm{m}$ where the change is 100\% (Li \& Draine 2001): since our distances are uncertain and we are only establishing a lower limit, the mass values estimated at $8 \mu \mathrm{m}$ are sufficient.
}

and third (after the XTE J0421+560/CI Cam system) HMXB systems to show mid-IR dust emission. Longer wavelength observations will be able to constrain the distribution and the total amount present, and ongoing IR spectroscopy (D. S. Moon et al. 2006, in preparation) will be able to probe the physical state of the dust particles. These discoveries, and the technique behind them, may serve as a pathfinder to probe the origins and properties of the newly discovered highly obscured hard X-ray binaries.

We thank an anonymous referee for comments that helped improve and clarify this paper. We also thank D. Chakrabarty and M. van Kerkwijk for help and advice. This work is based in part on archival data obtained with the Spitzer Space Telescope, which is operated by the JPL, Caltech, under NASA contract 1407. Support for this work was provided by NASA through an award issued by JPL/Caltech. This research has also made use of the data products from the Two Micron All Sky Survey, which is a joint project of the University of Massachusetts and the Infrared Processing and Analysis Center/Caltech, funded by NASA and NSF. D. S. M. was supported by a Millikan fellowship from Caltech; D. L. K. was supported in part by a fellowship from the Fannie and John Hertz Foundation.

Facilities: Spitzer(IRAC), MSX

\section{REFERENCES}

Belloni, T., et al. 1999, ApJ, 527, 345

Benjamin, R. A., et al. 2003, PASP, 115, 953

Boirin, L., et al. 2002, A\&A, 394, 205

Clark, J. S., Steele, I. A., Fender, R. P., \& Coe, M. J. 1999, A\&A, 348, 888

Cohen, M., Wheaton, W. A., \& Megeath, S. T. 2003, AJ, 126, 1090 (CWM03)

Coté, J., \& Waters, L. B. F. M. 1987, A\&A, 176, 93

Courvoisier, T. J.-L., et al. 2003, IAU Circ., 8063, 3

Cox, A. N. 2000, Allen's Astrophysical Quantities (4th ed.; New York: AIP)

Dale, D. A., et al. 2001, AJ, 122, 1736

Draine, B. T. 2003, ARA\&A, 41, 241

Fazio, G. G., et al. 2004, ApJS, 154, 10

Filliatre, P., \& Chaty, S. 2004, ApJ, 616, 469

Foschini, L., Rodriguez, J., \& Walter, R. 2003, IAU Circ., 8076, 2

Hildebrand, R. H., et al. 1977, ApJ, 216, 698

Indebetouw, R., et al. 2005, ApJ, 619, 931

Kaper, L., et al. 1995, A\&A, 300, 446

Kurucz, R. 1993, CD-ROM 13, ATLAS9 Stellar Atmosphere Programs and 2 $\mathrm{km} / \mathrm{s}$ Grid (Cambridge: SAO)

Lamers, H. J. G. L. M., et al. 1998, A\&A, 340, 117
Li, A., \& Draine, B. T. 2001, ApJ, 554, 778

Lutovinov, A., et al. 2005, A\&A, 433, L41

Matt, G., \& Guainazzi, M. 2003, MNRAS, 341, L13

Mukherjee, U., \& Paul, B. 2004, A\&A, 427, 567

Patel, S. K., et al. 2004, ApJ, 602, L45

Predehl, P., \& Schmitt, J. H. M. M. 1995, A\&A, 293, 889

Price, S. D., et al. 2001, AJ, 121, 2819

Revnivtsev, M. G. 2003, Astron. Lett., 29, 644

Revnivtsev, M., et al. 2003a, IAU Circ., 8097, 2

- 2003b, Astron. Lett., 29, 587

Skrutskie, M. F., et al. 2006, AJ, 131, 1163

Vidal, N. V. 1973, ApJ, 186, L81

Wagner, R. M., \& Starrfield, S. G. 1998, IAU Circ., 6857, 1

Walter, R., et al. 2003, A\&A, 411, L427

Waters, L. B. F. M., Coté, J., \& Geballe, T. R. 1988, A\&A, 203, 348

Werner, M. W., et al. 2004, ApJS, 154, 1

Winkler, C., et al. 2003, A\&A, 411, L349

Wright, A. E., \& Barlow, M. J. 1975, MNRAS, 170, 41 